\title{
Atualizações sobre a imunização contra o sarampo no Brasil: uma revisão sistemática
}

\author{
Updates on measles immunization in Brazil: a systematic review
}
Actualizaciones sobre la inmunización contra el sarampión en Brasil: una revisión sistemática

\begin{abstract}
Bruna Larissa Pinto Rodrigues ${ }^{1 *}$, Larissa Ribeiro de Souza ${ }^{1}$, Neiva Maria dos Santos Soares ${ }^{1}$, Karini de Nazaré Silva da Silva ${ }^{1}$, Aluísio Ferreira Celestino Júnior².
\end{abstract}

\section{RESUMO}

Objetivo: Analisar a produção científica acerca dos aspectos envolvidos no processo de imunização contra o sarampo no Brasil. Métodos: Estudo descritivo exploratório, realizado por meio de revisão sistemática da literatura, operacionalizada conforme o protocolo Preferred Reporting Itens for Systematic Reviews and MetaAnalyses (PRISMA). O levantamento de estudos bibliográficos foi realizado em bases de dados indexadas como LILACS, SciELO e MEDLINE. Resultados: O levantamento bibliográfico localizou 429 resultados. A análise das publicações resultou na amostra de 5 artigos, levantados pelos critérios estabelecidos. No período avaliado neste estudo, é possível identificar lacunas importantes na cobertura vacinal em vários locais do território nacional. As estratégias adotadas para enfrentamento do sarampo estão centradas em campanhas de vacinação, que apesar de serem comprovadamente eficazes, não têm tido a adesão necessária e há limitações que se enquadram na esteira de investimentos governamentais que poderiam impulsionar esta adesão. Considerações finais: As evidências permitiram identificar que a incompletude vacinal verificada tem como causa fatores diversos que precisam ser melhor dimensionados por novos estudos e devidamente enfrentados com estratégias consistentes que resultem em um controle mais rigoroso da enfermidade.

Palavras-chave: Sarampo, Imunização, Brasil, Vacina contra sarampo.

\begin{abstract}
Objective: Analyze the scientific production on the aspects involved in the measles immunization process in Brazil. Methods: Exploratory descriptive study, carried out by means of a systematic literature review, operationalized according to the Preferred Reporting Items for Systematic Reviews and Meta-Analyzes (PRISMA) protocol. The survey of bibliographic studies was carried out in indexed databases such as LILACS, SciELO and MEDLINE. Results: The bibliographic survey found 429 results. The analysis of the publications resulted in a sample of 5 articles, raised by the established criteria. In the period evaluated in this study, it is possible to identify important gaps in vaccination coverage in several places in the national territory. The strategies adopted to fight measles are centered on vaccination campaigns, which despite being proven to be effective, have not had the necessary adherence and there are limitations that fit in the wake of government investments that could impel this adherence. Final considerations: The evidence allowed to identify that the vaccine incompleteness verified is caused by several factors that need to be better dimensioned by new studies and properly dealt with consistent strategies that result in a stricter control of the disease.
\end{abstract}

Key words: Measles, Immunization, Brazil, Measles vaccine.

\footnotetext{
1 Universidade do Estado do Pará (UEPA). Belém - PA.

*E-mail: brunalarissa000@hotmail.com

2Universidade Federal do Pará (UFPA). Belém - PA
} 


\section{RESUMEN}

Objetivo: Analizar la producción científica sobre los aspectos involucrados en el proceso de inmunización contra el sarampión en Brasil. Métodos: Estudio exploratorio descriptivo, llevado a cabo mediante una revisión sistemática de la literatura, operacionalizado de acuerdo con el protocolo de ítems de informes preferidos para revisiones sistemáticas y metaanálisis (PRISMA). La encuesta de estudios bibliográficos se realizó en bases de datos indexadas como LILACS, SciELO y MEDLINE. Resultados: La encuesta bibliográfica encontró 429 resultados. El análisis de las publicaciones resultó en una muestra de 5 artículos, planteados por los critérios establecidos. En el período evaluado en este estudio, es posible identificar brechas importantes en la cobertura de vacunación en varios lugares del territorio nacional. Las estrategias adoptadas para combatir el sarampión se centran en las campañas de vacunación, que apesar de haberse demostrado que son efectivas, no han tenido la adherencia necesaria y existen limitaciones que se ajustan a las inversiones gubernamentales que podrían impulsar esta adhesión. Consideraciones finales: La evidencia permitió identificar que la incompletitud de la vacuna verificada es causada por varios factores que necesitan ser dimensionados mejor por nuevos estudios y tratados adecuadamente con estrategias consistentes que resulten en un control más estricto de la enfermedad.

Palabras clave: Sarampión, Inmunización, Brasil, Vacuna contra el sarampión.

\section{INTRODUÇÃO}

O sarampo é uma doença infectocontagiosa aguda, sistêmica, altamente transmissível e de notificação compulsória no Brasil desde 1968 (XAVIER AR, et al., 2019). É causado por um vírus pertencente à família Paramixoviridae e ao gênero Morbillivirus, possuindo três fases distintas: incubação, que dura de 10 a 14 dias, na qual o paciente é normalmente assintomático; prodrômica, que costuma durar de 2 a 4 dias, sendo o auge da transmissão; e exantemática, emergindo exantema maculopapular (sinais de Koplik). Suas manifestações clínicas incluem a presença de febre alta, mal-estar, tosse, coriza e conjuntivite, progredindo com diversas complicações como infecções respiratórias graves, encefalite, e eventualmente, óbito (MELLO JN, et al., 2014).

Segundo o Guia de Vigilância em Saúde, em 1992 o Brasil criou o Plano Nacional de Eliminação do Sarampo, formulando estratégias de vacinação, campanhas de divulgação e vigilância epidemiológica intensiva com a meta de erradicar a doença até o ano 2000. Com o progressivo decréscimo de incidência, em 2016 o Comitê Internacional de Especialistas (CIE) declarou a eliminação da circulação do vírus na região das Américas (BRASIL, 2017).

No entanto, a propagação do vírus em países na América do Sul e a baixa cobertura vacinal nestas populações determinaram a manutenção da doença no continente, sobretudo na Venezuela, que enfrenta desde 2017 um surto de sarampo. A atual situação sociopolítica e econômica da região ocasionou intenso movimento migratório que contribuiu para a circulação do vírus em regiões adjacentes, entre elas o Brasil (PEREIRA JPC, et al., 2019). De acordo com o boletim epidemiológico do Ministério da Saúde brasileiro, em 2019 foram confirmados 4.476 novos casos e 4 óbitos em 17 unidades federativas com transmissão ativa (BRASIL, 2019).

Convém destacar, ainda, que existem aspectos que influenciam diretamente a incidência, a evolução clínica e a letalidade do sarampo. O Ministério da Saúde ressalta que a doença afeta igualmente ambos os sexos, porém, fatores como as condições socioeconômicas, estado nutricional e defesa imunológica do doente podem ser determinantes (BRASIL, 2017).

Considerando que o tratamento consiste em suporte clínico, a vacinação é a medida profilática mais eficaz no manejo da doença em questão. Esta vacina foi introduzida no Brasil na década de 1960, e em 1973 incorporada à rotina de cuidados da criança com sua introdução no Programa Nacional de Imunização - PNI. Um dos principais objetivos desse programa é possibilitar uma drástica redução da morbimortalidade por doenças passíveis de prevenção por vacinação ao organizar, implementar e avaliar as ações de imunização 
em todo o país, ações que até então caracterizavam-se pela descontinuidade, pelo caráter episódico e pela reduzida área de cobertura (BRASIL, 2013).

No entanto, mesmo se houver interrupção da cadeia de transmissão autóctone do vírus, a vigilância e cobertura vacinal não podem ser negligenciadas (WALDMAN EA e SATO APS, 2016). Atualmente, é recomendado que pessoas na faixa entre 12 meses e 29 anos de idade recebam duas doses da vacina em intervalo de quatro semanas, e para a população entre 30 e 49 anos a indicação é uma dose. Existe contraindicação a alérgicos à neomicina ou a proteína do ovo (MELLO JN, et al., 2014).

Nos locais onde não há manutenção da homogeneidade nas coberturas vacinais, ou seja, inferiores a 95\% (nível considerado ideal pela OMS), a doença tende a apresentar comportamento endêmico. Esse aspecto depende diretamente da relação entre a imunidade e a suscetibilidade da população, assim como da circulação do vírus, que apresenta variação sazonal (BRASIL, 2017).

Levando em consideração a dimensão da doença em questão, seu potencial endêmico, bem como a necessidade de assegurar uma prática assistencial segura e baseada em evidências, o objetivo proposto deste artigo é analisar a produção científica acerca dos aspectos envolvidos no processo de imunização contra o sarampo no Brasil.

\section{MÉTODOS}

Estudo descritivo exploratório, realizado por meio de revisão sistemática da literatura. A presente investigação foi operacionalizada conforme o protocolo Preferred Reporting Itens for Systematic Reviews and Meta-Analyses (PRISMA), por meio das seguintes etapas: identificação, seleção, elegibilidade e inclusão (MOHER D, et al., 2015).

O levantamento de estudos bibliográficos foi realizado nas bases de dados indexadas: Literatura LatinoAmericana em Ciência de Saúde (LILACS), Medical Literature Analysis and Retrieval System Online (MEDLINE) e na Scientific Eletronic Library Online (SciELO). A seleção dos estudos foi realizada em duas buscas independentes, no período de novembro a dezembro de 2019. Os critérios de elegibilidade utilizados para seleção das publicações foram: estudos observacionais, originais e disponibilizados na íntegra, publicados nos idiomas inglês ou português no período compreendido entre 2014 e 2019. Nesse sentido, elegeu-se a seguinte pergunta norteadora para condução do estudo: Quais os aspectos relacionados ao panorama de imunização contra o Sarampo no Brasil?

Fazendo uso das seguintes palavras-chave encontradas nos Descritores em Ciências da Saúde (DeCs): "Sarampo", "Brasil", "Vacina contra Sarampo" e "Imunização", a busca foi feita de forma conjugada utilizando o operador booleano AND. Os critérios de exclusão foram artigos que se repetiam entre as bases de dados consultadas, que não respondiam aos objetivos propostos para este estudo, relatos de caso, estudos ecológicos, além de monografias, teses e cartas ao editor.

Após a triagem inicial, identificação dos estudos nas referidas bases de dados e aplicação dos critérios de inclusão, procedeu-se à leitura dos títulos e resumos, removendo as publicações que não atendiam à proposta da revisão, seguindo com a leitura na íntegra dos considerados elegíveis. Os artigos incluídos na revisão foram analisados criteriosamente, constatando na avaliação qualitativa que a metodologia empregada estava suficientemente descrita e adequada ao alcance dos objetivos propostos. Os estudos duplicados foram excluídos.

A avaliação da qualidade metodológica dos estudos incluídos foi efetuada mediante aplicação do instrumento - versão traduzida para o português (Brasil) - Strengthening the Reporting of Observational Studies in Epidemiology (STROBE). O checklist apresenta 22 itens e cada um dos critérios presentes recebeu uma pontuação de 0 a 1 , sendo o total transformado em percentual, considerando de boa qualidade os estudos primários superiores a 40\% (ALMEIDA MCV e CEZAR-VAZ MR, 2016; MALTA M, et al., 2010). Em relação à interpretação e síntese dos resultados, os artigos foram agrupados para facilitar a extração das informações, seguindo as semelhanças e comparando as diferentes abordagens dos estudos com 0 conhecimento teórico. Dessa forma, foi possível identificar conclusões e implicações resultantes da revisão. 


\section{RESULTADOS}

O levantamento bibliográfico localizou 429 resultados (Tabela 1), dos quais foram removidos 132 estudos duplicados. Após a leitura do título e do resumo, 275 publicações foram excluídas da revisão e 22 tornaramse elegíveis para leitura na íntegra. A análise das publicações pré-selecionadas resultou na amostra de 5 artigos científicos, levantados pelos critérios de inclusão estabelecidos. Seguindo a recomendação do grupo PRISMA, as estratégias de busca e razões para exclusão utilizadas nas respectivas bases de dados estão especificadas no fluxograma apresentado na Figura 1 (MOHER D, et al., 2015).

Tabela 1 - Publicações dos anos de 2014 a 2019 sobre imunização contra o Sarampo no Brasil.

\begin{tabular}{lccc}
\hline \multicolumn{1}{c}{ Cruzamentos } & \multicolumn{3}{c}{ Bases de dados } \\
\hline Sarampo AND Imunização AND Brasil & SciELO & LILACS & MEDLINE \\
Sarampo AND Imunização & 7 & 7 & 7 \\
Sarampo AND Brasil & 9 & 3 & 7 \\
Brazil AND Imunização & 8 & 8 & 10 \\
Vacina contra sarampo AND Brasil & 63 & 90 & 194 \\
\hline Total: 429 & 7 & 3 & 6 \\
\hline
\end{tabular}

Fonte: Rodrigues BLP, et al., 2020.

Figura 1 - Fluxograma baseado no protocolo PRISMA sobre a seleção de evidências.

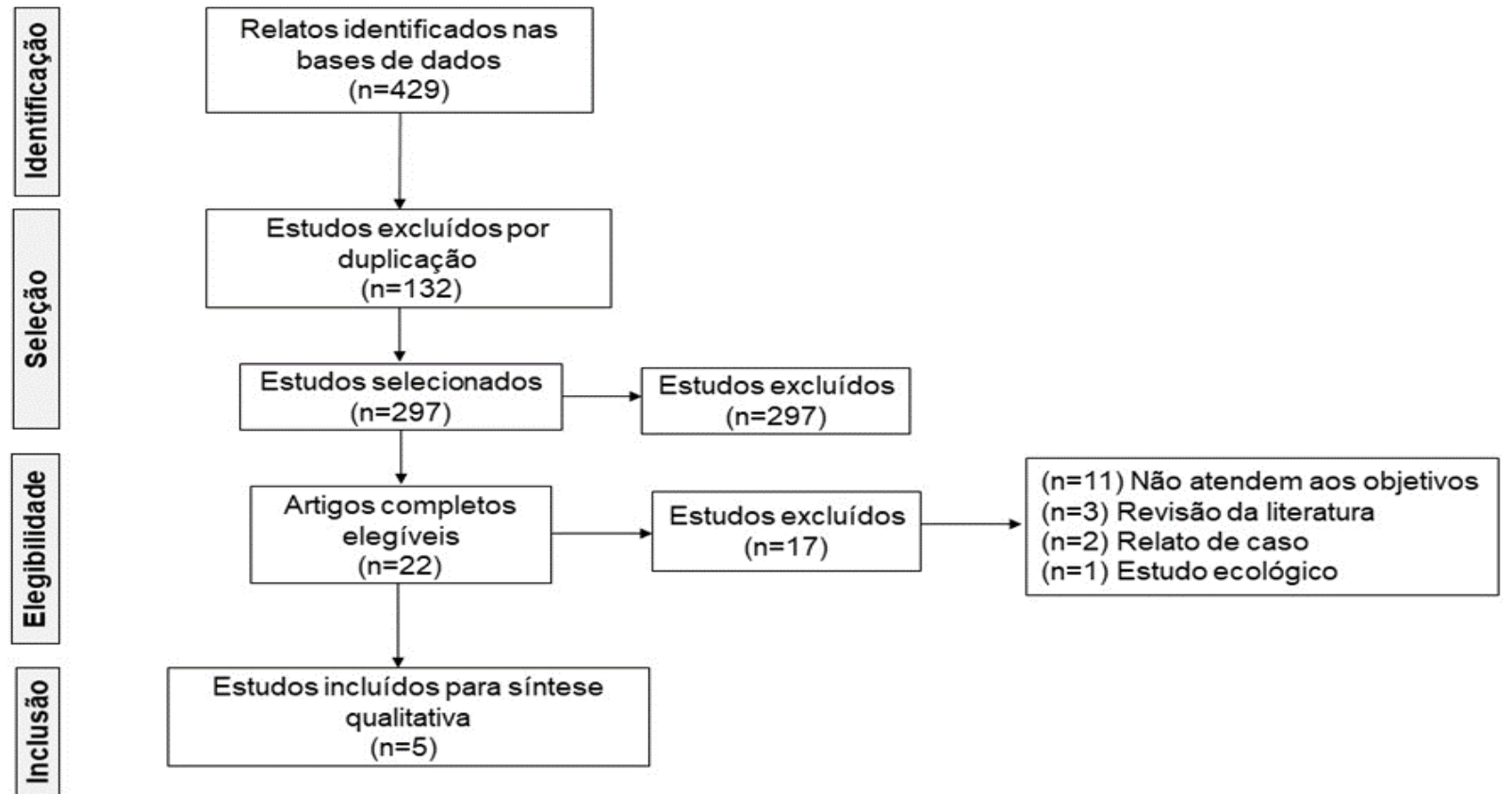

Fonte: Rodrigues BLP, et al., 2020.

Conforme apresentado na Tabela 2, observa-se uma distribuição temporal linear na divulgação dos artigos, todos publicados entre os anos de 2015 e 2019, sinalizando um aumento de interesse nas investigações de surto de sarampo, bem como nas estratégias de imunização adotadas para enfrentamento da patologia. A maioria das referências selecionadas são voltadas para as regiões Norte e Nordeste do Brasil. Em relação ao idioma, a maior parte $(80 \%)$ das produções foram encontradas na língua portuguesa. A divulgação científica ocorreu em 4 periódicos, a saber: Ciência \& Saúde Coletiva, Epidemiol. Serv. Saúde, Cad. Saúde Pública e Journal Vaccine. Em relação à característica metodológica, todos os estudos primários apresentaram boa qualidade (>40\%) em relação aos itens propostos na lista de verificação (checklist) STROBE para estudos observacionais (Tabela 2). 


\section{Revista Eletrônica Acervo Saúde / Electronic Journal Collection Health | ISSN 2178-2091}

Tabela 2 - Caracterização dos estudos primários incluídos na revisão sistemática.

\begin{tabular}{|c|c|c|c|c|}
\hline Título & $\begin{array}{l}\text { Autor/Ano de } \\
\text { publicação }\end{array}$ & $\begin{array}{c}\text { Delineamento do } \\
\text { estudo }\end{array}$ & Objetivo & $\begin{array}{c}\text { STROBE } \\
(\%)\end{array}$ \\
\hline $\begin{array}{l}\text { A vacinação e o saber do } \\
\text { adolescente: educação em saúde e } \\
\text { ações para imunoprevenção. }\end{array}$ & $\begin{array}{l}\text { (VIEGAS SMF, et } \\
\text { al., 2019) }\end{array}$ & Transversal & $\begin{array}{c}\text { Analisar a situação vacinal de adolescentes do 9a ano do ensino } \\
\text { fundamental de escolas públicas de Divinópolis-MG e o conhecimento } \\
\text { sobre as doenças transmissíveis e as imunopreveníveis. }\end{array}$ & $90,90 \%$ \\
\hline $\begin{array}{l}\text { Incompletude vacinal infantil de } \\
\text { vacinas novas e antigas e fatores } \\
\text { associados: coorte de Nascimento } \\
\text { BRISA, São Luís, Maranhão, } \\
\text { Nordeste do Brasil. }\end{array}$ & $\begin{array}{c}\text { (SILVA MC, et al., } \\
\text { 2018) }\end{array}$ & Coorte prospectivo & $\begin{array}{l}\text { Estimar percentuais de incompletude para vacinas do Calendário } \\
\text { Nacional de Vacinação da Criança, para o primeiro ano de vida e } \\
\text { fatores associados a eles, tanto para o esquema vacinal para novas } \\
\text { vacinas (EVNV) quanto para o esquema vacinal para antigas vacinas } \\
\text { (EVAV), em crianças de } 13 \text { a } 35 \text { meses de idade. }\end{array}$ & $100 \%$ \\
\hline $\begin{array}{l}\text { Coberturas vacinais por doses } \\
\text { recebidas e oportunas com base } \\
\text { em um registro informatizado de } \\
\text { imunização, Araraquara-SP, Brasil, } \\
\text { 2012-2014. }\end{array}$ & $\begin{array}{l}\text { (TAUIL FS, et al., } \\
\text { 2017) }\end{array}$ & Coorte descritivo & $\begin{array}{l}\text { Descrever as coberturas vacinais por tipo de vacina aos } 12 \text { e aos } 24 \\
\text { meses de idade, no município de Araraquara, estado de São Paulo, } \\
\text { Brasil. }\end{array}$ & $86,36 \%$ \\
\hline $\begin{array}{l}\text { Factors associated with non- } \\
\text { vaccination against measles in } \\
\text { northeastern Brazil: Clues about } \\
\text { causes of the } 2015 \text { outbreak. }\end{array}$ & $\begin{array}{l}\text { (ROCHA HAL, et } \\
\text { al., 2015) }\end{array}$ & Transversal & $\begin{array}{l}\text { Identificar os fatores determinantes da não vacinação no Brasil em dois } \\
\text { momentos diferentes de cobertura vacinal, para fornecer pistas sobre } \\
\text { as causas dos surtos atuais. }\end{array}$ & $100 \%$ \\
\hline $\begin{array}{l}\text { Investigação de surto de sarampo } \\
\text { no Estado do Pará na era da } \\
\text { eliminação da doença no Brasil. }\end{array}$ & $\begin{array}{l}\text { (JESUS HS, et } \\
\text { al., 2015) }\end{array}$ & $\begin{array}{l}\text { Coorte } \\
\text { retrospectivo }\end{array}$ & $\begin{array}{l}\text { Investigar casos de sarampo no estado do Pará, confirmar a existência } \\
\text { do surto, identificar genótipo do vírus circulante e recomendar medidas } \\
\text { de controle e prevenção. }\end{array}$ & $72,72 \%$ \\
\hline
\end{tabular}

Fonte: Rodrigues BLP, et al., 2020. 


\section{DISCUSSÃO}

O propósito desta revisão foi buscar na literatura científica o panorama das Imunizações no Brasil e o que influencia na efetividade das estratégias implementadas. A maioria dos estudos selecionados aponta padrões distintos de determinantes sociais de saúde e que os fatores associados aos serviços e programas de imunização são conduzidos pelo contexto, sendo sua compreensão importante na ampliação da cobertura vacinal do Sarampo (TAUIL FS, et al., 2017; SILVA MC, et al., 2018; ROCHA HAL, et al., 2015; JESUS HS, et al., 2015).

Apesar de diferenças quanto à abrangência geográfica, períodos analisados e metodologia estatística, foi possível constatar cobertura abaixo da meta estabelecida pelo Ministério da Saúde para SCR (Sarampo, Caxumba e Rubéola), o que implica em mortalidade significativamente maior, especialmente em casos de surtos (TAUIL FS, et al., 2017; BRASIL, 2020).

A vigilância à saúde constitui-se como um pilar fundamental na prevenção e proteção contra infecções que possuem grau de disseminação elevado, como a que o sarampo apresenta (MELLO JN, et al., 2014). Esta enfermidade, além do alto grau de propagação, ainda é motivo de preocupação por sua letalidade. Estas características seriam suficientes para manter as estratégias de prevenção que o Brasil adotou no passado, mas que nos últimos anos foram negligenciadas, resultando em exposição de suscetíveis ao vírus que propiciaram a reemergência da doença (LUNA EJA e SILVA JB Jr., 2013).

O Brasil vem enfrentando grandes surtos de sarampo em consonância com o decréscimo das coberturas vacinais observadas a partir de 2016 (SATO APS, 2018). Essa situação demonstra que a presença de bolsões de pessoas não vacinadas, seja proveniente de razões filosóficas ou falta de acesso a cuidados de saúde, pode sustentar a transmissão do sarampo no território (JESUS HS, et al., 2015).

De acordo com o Ministério da Saúde, no ano de 2019 foram confirmados 18.203 casos de sarampo no país. A faixa etária de 20 a 29 anos permaneceu com um elevado número de não vacinados, sendo justamente esta, a faixa etária responsável pela ocorrência do maior número de casos. Dessa forma, atingir elevada cobertura vacinal na população nesse público tem grande potencial para evitar casos nas crianças, em especial nas menores de 6 meses de idade, grupo este com contraindicação à vacinação e mais suscetível a complicações e óbitos por sarampo (BRASIL, 2020).

No período avaliado neste estudo, é possível identificar lacunas importantes na cobertura vacinal em vários locais do território nacional (JESUS HS, et al., 2015; ROCHA HAL, et al., 2015; SILVA FS, et al., 2018; VIEGAS SMF, et al., 2019). Tais locais, em termos epidemiológicos, não representam uma amostra fidedigna para redundar em uma generalização consistente para todo o país, mas já apontavam para um risco de reintrodução da doença em território nacional com consequências relativamente previsíveis. $O$ estudo de Rocha HAL, et al. (2015) destaca que a cobertura vacinal baixa no Brasil não é um fenômeno recente. Em 1987 já se verificara uma cobertura inferior a $50 \%$, o que deixou um grupo significativo de pessoas sem proteção. Tal grupo, em grande parte, representa parcela dos indivíduos suscetíveis a essa infecção atualmente.

A queda nas coberturas vacinais e suas consequências visíveis justificam os esforços para melhor compreender a hesitação vacinal no Brasil (SATO APS, 2018). No entanto, apesar do impacto na redução de casos e mortes pelas doenças imunopreveníveis, movimentos antivacinação são cada vez mais frequentes e persuasivos, utilizando estratégias de distorção e divulgação de informações falsas que alegam embasamento científico, questionam a eficácia e segurança de diversas vacinas (APS LRMM, et al., 2018). Tais relatos, em sua maioria, relacionam vacinas como a tríplice viral, adjuvantes e o conservante timerosal com a ocorrência de síndrome de espectro autista em crianças (VASCONCELLOS-SILVA PR, et al., 2015).

A adesão ao Movimento Antivacina preocupa o Ministério da Saúde à medida que provoca quedas no índice de cobertura de alguns imunizantes oferecidos pelo Sistema Único de Saúde (SUS) (SHIMIZU NR, 2018). O ano de 2017 teve o menor índice, em 16 anos, de cobertura vacinal em crianças e apenas $69,95 \%$ da população tomou a segunda dose da vacina contra o sarampo, quando a meta estipulada era de $95 \%$. (BRASIL, 2019). 
Segundo Viegas SMF, et al. (2019), um fator que pode estar associado à baixa cobertura vacinal é a falta de conhecimento referente às vacinas e sobre as doenças transmissíveis e imunopreveníveis. No estado do Piauí foi realizada uma pesquisa com 44.157 adolescentes, na faixa etária de 10 a 19 anos e foi constatado que $60,2 \%$ desconhecia o calendário vacinal (CARVALHO AMC e ARAÚJO TME, 2012). Por outro lado, foi observado que a administração da vacina nas escolas está associada a maior completude do calendário vacinal dos adolescentes, levando em consideração a baixa procura dessa população pelas unidades de saúde (GALLAGHER KE, et al., 2016).

De acordo com Rocha HAL, et al. (2015), em um contexto de baixa cobertura, os principais fatores determinantes da não vacinação infantil contra o sarampo estão relacionados com a dificuldade de acesso aos serviços, a alimentação e nutrição das crianças, além de fatores socioeconômicos, representados pelo baixo nível de escolaridade da mãe e pelas condições desfavoráveis do abastecimento de água e saneamento. Neste estudo, crianças de famílias com dificuldades de acesso a uma instalação de saúde, água corrente e esgotos tinham 23 a $38 \%$ mais probabilidades de não serem vacinadas contra o sarampo do que as crianças com acesso. Além disso, a mãe analfabeta também aumentou, em 19\%, o risco de não vacinação.

Neste ponto os autores corroboram com achados no estudo de Silva BS et al. (2018) que identificou fatores comportamentais e reprodutivos envolvidos, enfatizando a importância de atentar para a vulnerabilidade de filhos de mães adolescentes, tabagistas, que não planejaram a gravidez, engravidaram no primeiro ano após o nascimento da criança em estudo, realizaram menos de seis consultas pré-natais ou iniciaram no terceiro trimestre.Resultado semelhante foi encontrado em uma pesquisa realizada por Oliveira MFS, et al. (2014), revelando que há uma tendência decrescente da cobertura vacinal relacionada ao fato de ser membro de famílias numerosas e de classes socioeconômicas menos favorecidas, acarretando aumento da suscetibilidade de não cumprimento dos esquemas vacinais pela presença de mecanismos que comprometem o acesso a bens e serviços.

Outro fator que pode influenciar de forma decisiva a efetividade das estratégias de vacinação são as barreiras de acesso aos serviços de saúde. Silva ZP, et al. (2011), apontaram que a dificuldade em conseguir atendimento, o desabastecimento e a descontinuidade no serviço de vacinação podem levar a oportunidades perdidas de vacinação e afetar o cumprimento do calendário vacinal. Outro estudo que buscou avaliar a percepção de usuários sobre o acesso à vacinação na atenção primária mostrou que a distância da APS, ausência de transporte coletivo e barreiras no trajeto foram apontados como dificultadores do acesso. A indisponibilidade de alguns imunobiológicos também foi descrita como um aspecto que gerou insatisfação aos usuários, que por limitações financeiras, não têm acesso às vacinas ofertadas no mercado privado (DUARTE DC, et al., 2018).

As estratégias adotadas pelo Ministério da Saúde para o controle do sarampo estão centradas na realização de Campanhas de Vacinação, cuja ação é eficaz, segura e seu acesso é universal, tendo em vista a disponibilidade das vacinas nas unidades básicas de saúde em todos os municípios brasileiros. Apesar de serem comprovadamente eficazes, não têm tido a adesão necessária e há limitações que se enquadram na esteira de investimentos governamentais que poderiam impulsionar esta adesão. Dentre estes investimentos enquadram-se o melhor aparelhamento da atenção básica e da mobilização da população através de recursos de comunicação, sensibilizando o público específico onde a vacinação deve ser priorizada (COUGHLIN M, et al., 2017).

Em resposta ao surto ocorrido no Brasil em 2019 foi adotada uma série de iniciativas para controle da doença, tais como: a busca ativa dos casos, o bloqueio vacinal dos contatos, a atualização de cartão de vacina e campanhas de mídia para conscientização da população (BRASIL, 2020). Por tais razões oportunas, é evidentemente necessária a condução de ações de vacinação de maneira massiva para possibilitar maior proteção à população quanto as doenças imunopreveníveis. O estado do Ceará apresentou resultados de cobertura vacinal superiores a 95\% no enfrentamento da epidemia de Sarampo em 2014, empregando estratégias que incluem a intensificação em postos móveis e varredura, com a captação comunitária de não vacinados e suscetíveis, a convocatória dos gestores locais, municipais e estaduais, além da formação de equipes de resposta rápida (MOURA ADA, et al., 2018).

REAS/EJCH | Vol.Sup.n.55 | e3919 | DOI: https://doi.org/10.25248/reas.e3919.2020 Página 7 de 9 
O desafio de se obter uma alta cobertura vacinal (>95\%), deve ser uma meta sempre desejável, como também estar sempre associada aos aspectos operacionais que a viabilizem. Tal cobertura garante uma redução significativa dos suscetíveis e alcança os indivíduos que podem desenvolver os casos com maiores complicações (FERNANDES EG, et al., 2013). O monitoramento dessas coberturas vacinais é essencial para se verificar se os parâmetros estão sendo alcançados (SILVA BS, et al., 2018). Um estudo com base no Registro Informatizado de Imunização (RII) observou percentuais mais baixos de vacinação em relação às doses oportunas, sendo 53,5\% a taxa para Sarampo, Caxumba e Rubéola, revelando o atraso vacinal.

O RII mostrou-se relevante para monitoramento de coberturas vacinais, com análises mais acuradas que permitem um bom aproveitamento das informações (SATO APS, 2015). No entanto, apesar das vantagens dos registros eletrônicos de saúde, estudos têm apontado dificuldades para sua operacionalização. Neste ponto destaca-se a heterogeneidade de qualidade na completude e precisão dos dados, além da fragmentação nos cuidados de saúde, uma vez que demanda além aquisição e manutenção da tecnologia, mudança organizacional, incentivo financeiro e padrões de interoperabilidade (SILVA FS, et al., 2018).

A notificação compulsória da infecção foi de grande relevância para uma melhor dimensão e controle da infecção. Entretanto, é necessário agilidade no fluxo da informação epidemiológica, haja vista que a notificação tardia pode influenciar negativamente o controle da cadeia de transmissão e, por esta razão, dificultar a implementação das estratégias de controle (BRASIL, 2019).

Antes da circulação sustentada do vírus em determinada região, é possível identificar quais são os genótipos virais circulantes nos indivíduos infectados. Casos importados ou casos de reemergência de genótipos locais tem significado distinto para os operadores do sistema de saúde (ROCHA HAL, et al., 2015), notadamente aqueles que poderão antever ameaças como as que envolvem a circulação de pessoas em eventos artísticos, políticos, religiosos, esportivos, etc.

Enfermidades como o sarampo tem potencialidade de circulação crítica em virtude de sua alta transmissibilidade e isso se agrava em contextos cada vez mais globalizados por onde circula. Desta forma, justifica-se ainda mais o alcance da meta de cobertura vacinal acima dos 95\% (SILVA FS, et al., 2018).

Considerando este cenário, há que se ensejar iniciativas mais consistentes de enfrentamento desta enfermidade e, a considerar a literatura levantada, tem potencial de gerar um número maior de infectados, dentre os quais alguns irão evoluir para complicações, além de aumentar os custos do sistema de saúde no âmbito da assistência de alta complexidade.

\section{CONSIDERAÇÕES FINAIS}

O estudo demonstrou que a circulação sustentada do vírus do sarampo no Brasil nos últimos anos, teve como principal razão a baixa cobertura vacinal de sua população. Mesmo tendo genótipos importados como a principal ameaça, um expressivo número de pessoas suscetíveis permitiu que a situação de disseminação do vírus tomasse proporção extremamente preocupantes. Sendo assim, é fundamental que mais pesquisas voltadas para essa problemática social de saúde sejam realizadas, no sentido de avaliar o índice de cobertura vacinal e melhor dimensionar os fatores que contribuem para sua incompletude, visto que inseguranças, desconhecimento sobre o imunobiológico e a falta de estratégias consistentes de captação e orientação pelas equipes de saúde contribuem para a perda de oportunidade vacinal.

\section{REFERÊNCIAS}

1. ALMEIDA CCC, et al. Estudo epidemiológico de pacientes infectados por Sarampo no Brasil, Brazilian. Journal of Health Review, 2020; 3(2):1513-1526.

2. ALMEIDA MCV, CEZAR-VAZ MR. Scientific evidence of dockworker illness to nursing clinical reasoning. Revista da Escola de Enfermagem, 2016; 50(2): 341-349.

3. APS LRMM, et al. Eventos adversos de vacinas e as consequências da não vacinação: uma análise crítica, Brasil. Revista de Saúde Pública, 2018; 52: 40.

4. BRASIL, MS. Secretaria de Vigilância em Saúde. Coordenação geral de Desenvolvimento da Epidemiologia em 
Serviços. Guia de Vigilância em Saúde, 2017; único: 01-706.

5. BRASIL, MS. Secretaria de Vigilância em Saúde. Coordenação Geral do Programa Nacional de Imunizações. Boletim Epidemiológico- Cobertura nacional da vacina tríplice viral: primeira dose para crianças com até 1 ano, de 2015 a 2019, 2019; 50(29).

6. BRASIL, MS. Secretaria de Vigilância em Saúde. Departamento de Imunização e Doenças Transmissíveis. Boletim Epidemiológico. Vigilância Epidemiológica do Sarampo no Brasil 2019: janeiro a dezembro, 2020; 51(06): 01-37.

7. BRASIL, MS. Secretaria de Vigilância em Saúde. Departamento de Vigilância Epidemiológica. Programa Nacional de Imunizações (PNI): 40 anos, 2013. 1: 01-228.

8. CARVALHO AMC, ARAÚJO TME. Conhecimento do adolescente sobre vacinano ambiente da Estratégia Saúde da Família. Revista Brasileira de Enfermagem, 2012; 65(2): 229-235.

9. COUGHLIN M, et al. Perspective on Global Measles Epidemiology and Control and the Role of Novel Vaccination Strategies, United States. Viruses, 2017; 9(1): 11.

10. DUARTE DC, et al. Acesso à vacinação na Atenção Primária na voz do usuário: sentidos e sentimentos frente ao atendimento, Brasil. Escola Anna Nery, 2019; 23(1): 1-8.

11. FERNANDES EG, et al. Surto de sarampo na regiao metropolitana de Campinas-SP, Brasil. Revista de Saúde Pública, 2013; 47(6): 1213-1217.

12. GALLAGHER KE, et al. Factors influencing completion of multidose vaccine schedules in adolescents: a systematic review, United Kingdom. BMC Public Health, 2016; 16:172.

13. JESUS HS, et al. Investigação de surto de sarampo no Estado do Pará na era da eliminação da doença no Brasil. Cadernos de Saúde Pública, 2015; 31(10): 2241-2246.

14. LUNA EJA, SILVA JR. JB. Doenças transmissíveis, endemias, epidemias e pandemias. A saúde no Brasil em 2030 : população e perfil sanitário. Fiocruz/lpea/Ministério da Saúde/Secretaria de Assuntos Estratégicos da Presidência da República, 2013; 2:123-176.

15. MALTA M, et al. STROBE initiative: guidelines on reporting observational studies, Brasil. Revista de Saúde Pública, 2010; 44(3): 559-565.

16. MELLO JN, et al. Panorama atual do sarampo no mundo: Risco de surtos nos grandes eventos no Brasil. Jbm, 2014; 102 (1): 33-40.

17. MOHER D, et al. Principais itens para relatar Revisões sistemáticas e Meta-análises: A recomendação PRISMA, Brasil. Epidemiologia e Serviços de Saúde, 2015; 24(2): 335-342.

18. MOURA ADA, et al. Estratégias e resultados da vacinação no enfrentamento da epidemia de sarampo no estado do Ceará, 2013-2015, Brasil. Epidemiologia e serviços de saúde, 2018; 27(1):1-8.

19. OLIVEIRA MFS, et al. Fatores associados à cobertura vacinal em menores de cinco anos em Angola. Revista de Saúde Pública, 2014; 48(6): 906-915.

20. PEREIRA JPC, et al. Negligência à vacinação: o retorno do Sarampo ao Brasil. Anais da I JORNADA CAMED UNIBH 29, 2019; 12(1): 1-5.

21. ROCHA HAL, et al. Factors associated with non-vaccination against measles in northeastern Brazil: Clues about causes of the 2015 outbreak. Vaccine, 2015; 33(38): 4969-4974.

22. SATO APS. National Immunization Program: Computerized System as a tool for new challenges. Revista de Saúde Pública, 2015; 49: 1-5.

23. SATO APS. What is the importance of vaccine hesitancy in the drop of vaccination coverage in Brazil? Revista de Saúde Pública, 2018; 52:1-9.

24. SHIMIZU NR, Movimento Antivacina: A memória funcionando no/pelo (per)curso dos sentidos e dos sujeitos na sociedade e-urbana. Revista do EDICC, 2018; 5(1): 87-97.

25. SILVA BS, et al. Evaluation study of the National Immunization Program Information System. Revista Brasileira de Enfermagem, 2018; 71(1): 615-624.

26. SILVA FS, et al. Incompletude vacinal infantil de vacinas novas e antigas e fatores associados: Coorte de nascimento BRISA, São Luís, Maranhão, Nordeste do Brasil. Cadernos de Saúde Pública, 2018; 34(3): 2-21.

27. SILVA ZP, et al. Perfil sociodemográfico e padrão de ultilização dos serviços de saúde do Sistema Único de Saúde (SUS), 2003-2008, Brasil. Ciência \& Saúde Coletiva, 2011; 16(9): 3807-3816.

28. TAUIL MC, et al. Coberturas vacinais por doses recebidas e oportunas com base em um registro informatizado de imunização, Araraquara-SP, Brasil, 2012-2014. Epidemiologia e Serviços de Saúde, 2017; 26(4): 835-846.

29. VASCONCELLOS-SILVA PR, et. al. A sociedade de risco midiatizada, o movimento antivacinação e o risco do autismo, Brasil. Ciência \& Saúde Coletiva, 2015; 20(2): 607-616.

30. VIEGAS SMF, et al. A vacinação e o saber do adolescente: educação em saúde e ações para a imunoprevenção, Brasil. Ciência \& Saúde Coletiva, 2019; 24(2): 351-360.

31. WALDMAN EA, SATO APS. Path of infectious diseases in Brazil in the last 50 years: an ongoing challenge. Revista de Saúde Pública, 2016; 50: 1-18.

32. XAVIER AR, et al. Diagnóstico clínico, laboratorial e profilático do sarampo no Brasil. Jornal Brasileiro de Patologia e Medicina Laboratorial, 2019; 55 (4): 396-401. 